\title{
Delivery of microRNAs by Chitosan Nanoparticles to Functionally Alter Macrophage Cholesterol Efflux in Vitro and in Vivo
}

My-Anh Nguyen ${ }^{1}$, Hailey Wyatt ${ }^{1}$, Leah Susser ${ }^{1}$, Michelle Geoffrion ${ }^{1}$, Adil Rasheed ${ }^{1}$, Anne-Claire Duchez ${ }^{1}$, Mary Lynn Cottee ${ }^{1}$, Esther Afolayan ${ }^{2}$, Eliya Farah ${ }^{2}$, Zaina Kahiel ${ }^{2}$, Marceline Côté ${ }^{2}$, Suresh Gadde ${ }^{2^{*}}$, Katey J Rayner ${ }^{1,2^{*}}$

${ }^{1}$ University of Ottawa Heart Institute, Ottawa, Ontario K1Y 4W7, Canada

2Department of Biochemistry, Microbiology and Immunology, Faculty of Medicine, University of Ottawa, Ottawa, Ontario K1H 8M5, Canada

${ }^{*}$ Correspondence to:

Suresh Gadde: sgadde@uottawa.ca or Katey J Rayner: krayner@ottawaheart.ca 

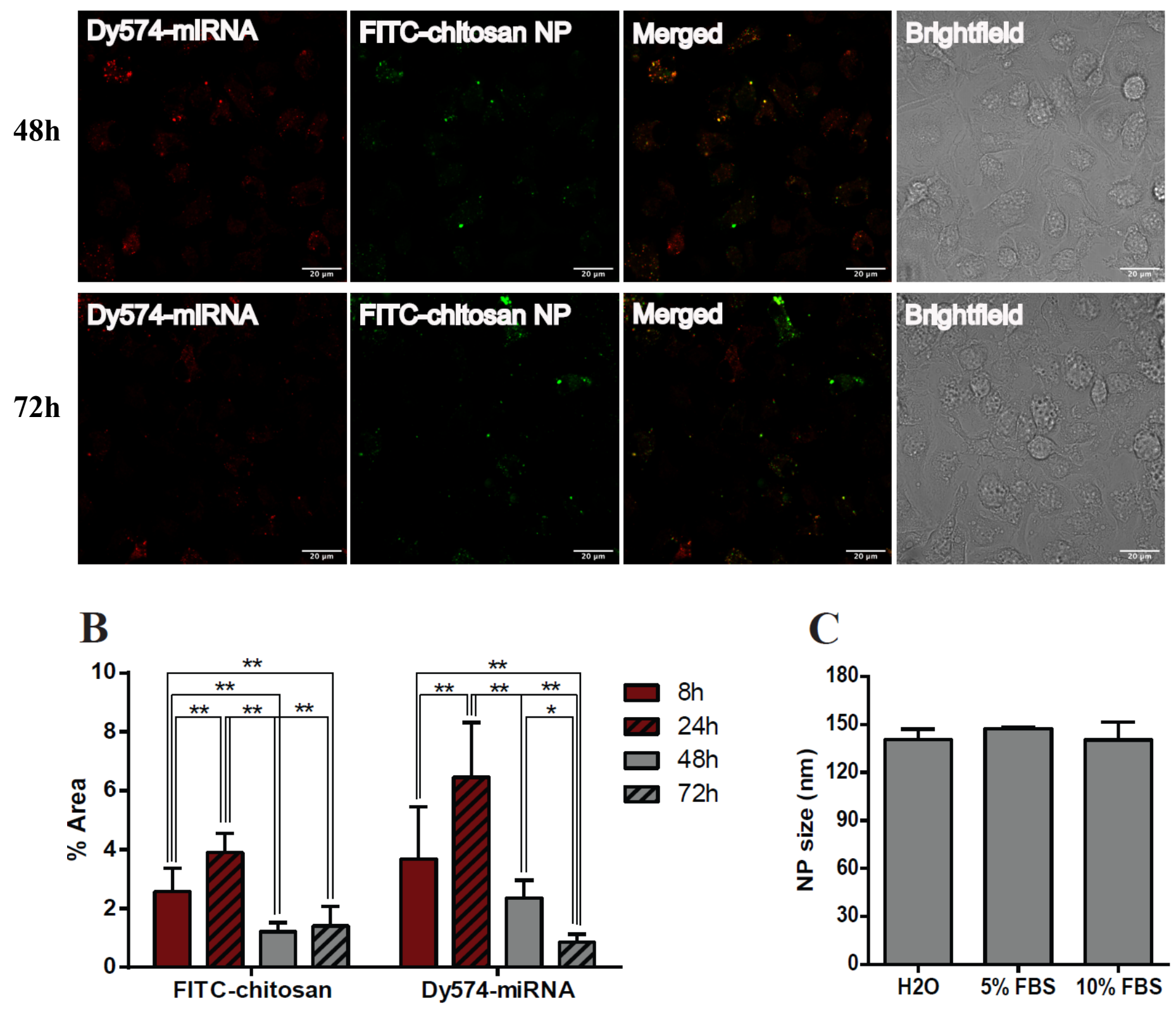

Supplemental Figure 1: Chitosan nanoparticles containing miRNA mimics are taken up and deliver exogenous miRNAs to naïve macrophages. (A) FITC-labeled chitosan nanoparticles containing Dy547-labeled miRNA mimics were prepared and applied to peritoneal macrophages. Uptake of labeled nanoparticles and miRNA mimics was visualized using confocal microscopy. Scale bar s represent $20 \mu \mathrm{m}$. (B) The area of fluorescent signal from (A) was quantified using Image $\mathrm{J}$ software using 4 fields for each condition. Graph represents the mean $\pm S D$ from $n=3$ independent experiments. (C) Chitosan nanoparticles containing cel-miR-54 were incubated $\mathrm{H}_{2} \mathrm{O}, \mathrm{DMEM}$ supplemented with $5 \%$ FBS, or DMEM supplemented with $10 \%$ FBS. The size of nanoparticles were measured preand $2 \mathrm{~h}$ post incubation using Malvern Zetasizer. 
A

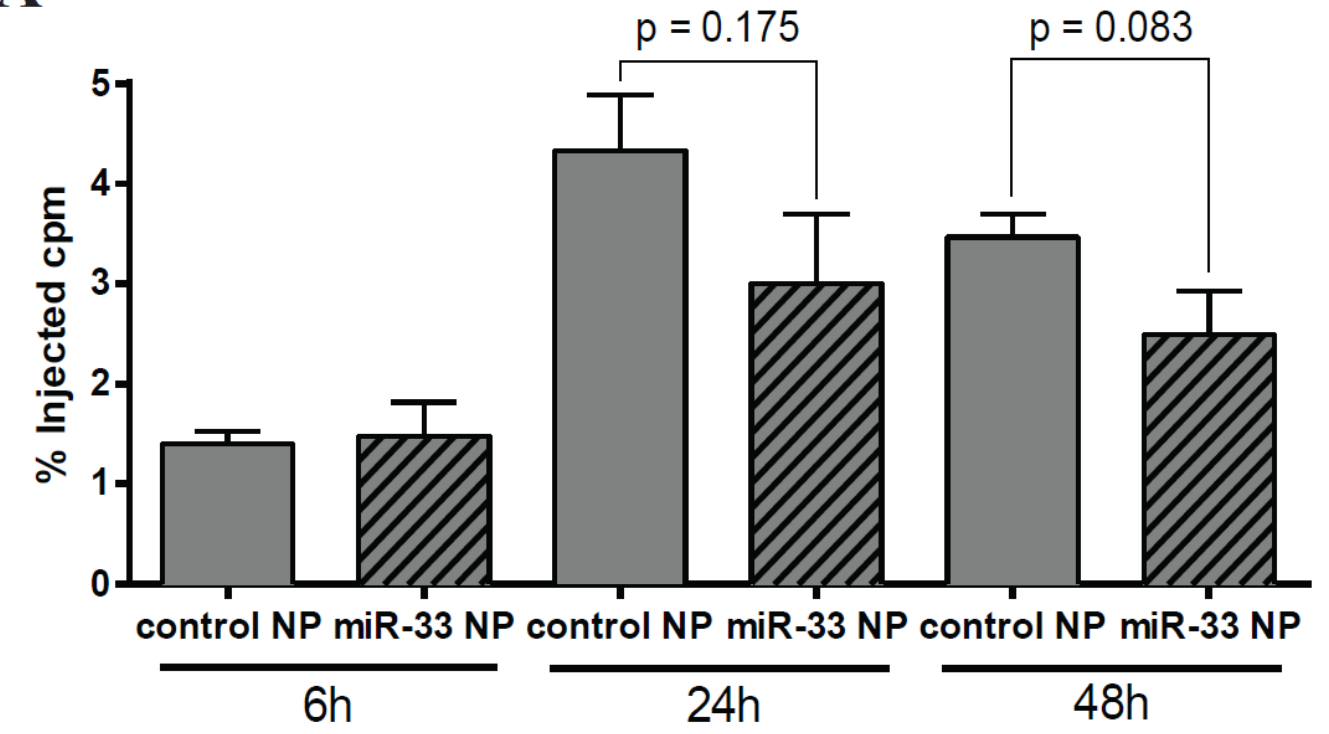

B

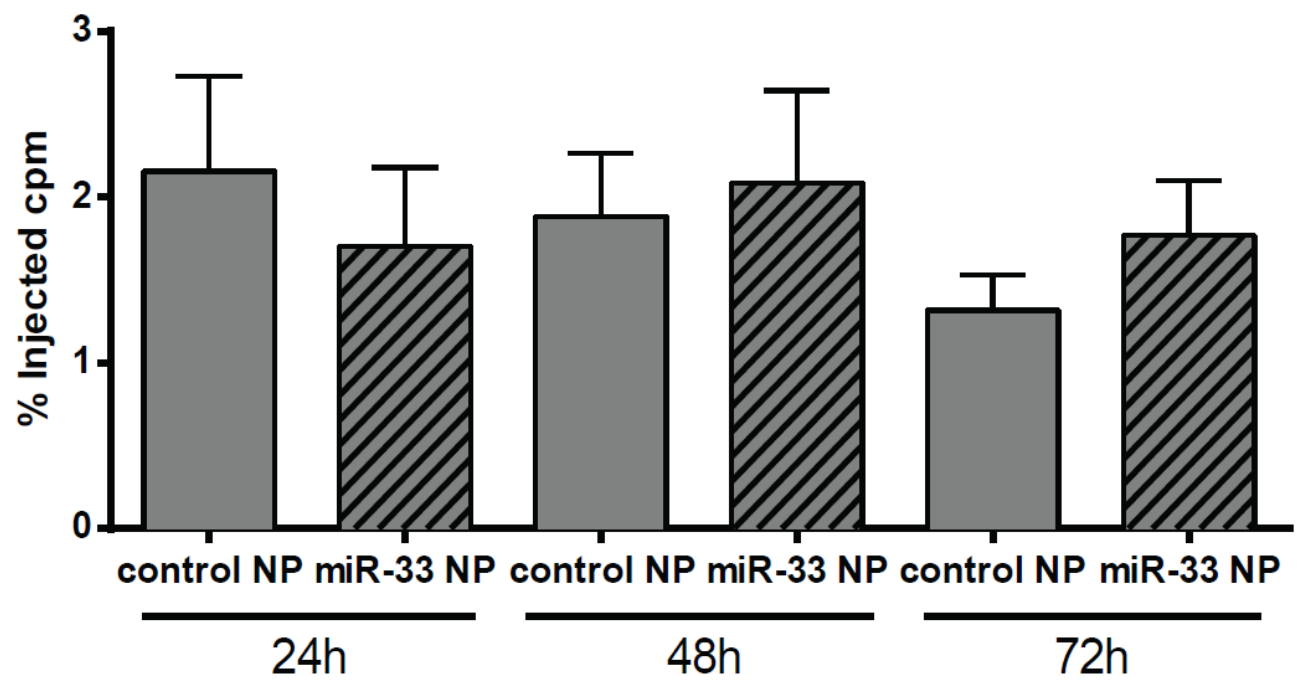

Supplemental Figure 2: Time course of ${ }^{3} \mathrm{H}$-cholesterol distribution in plasma. C57BL6 mice ( $\left.n=5 / g r o u p\right)$ were injected subcutaneously with $(\mathbf{A})$ acLDL-loaded/[ $\left.{ }^{3} \mathrm{H}\right]$ cholesterol-labeled peritoneal macrophages pre-treated with control/miR-33 nanoparticles for $48 \mathrm{~h}$ or (B) acLDL-loaded/[ $\left.{ }^{3} \mathrm{H}\right]$ cholesterol-labeled peritoneal macrophages and chitosan nanoparticles containing control/miR-33 mimics. Data are expressed as the percentage of the ${ }^{3} \mathrm{H}$-cholesterol tracer relative to that of total $\mathrm{cpm}$ tracer injected $\pm \mathrm{SD}$. 